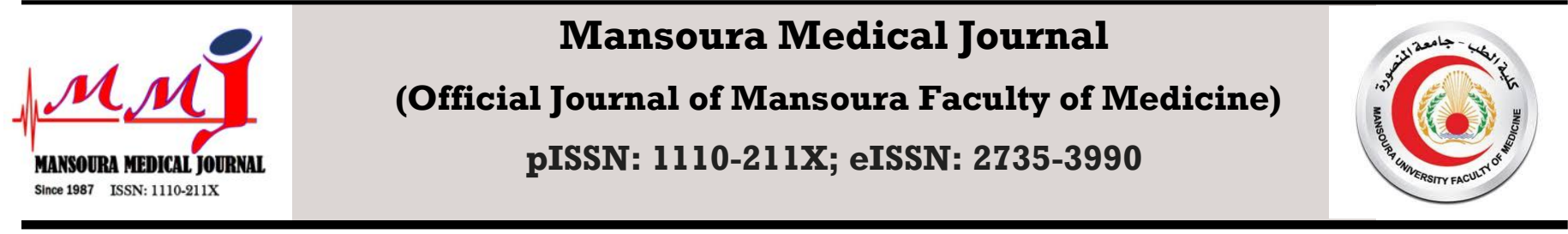

\title{
Characteristics of type 2 Diabetes mellitus in Egyptian Elderly Population By
}

\author{
Tahia Abdel-Rahman ${ }^{1}$, Mohamed A. Helaly ${ }^{2}$, Mohamed El-Arman ${ }^{3}$,
}

\author{
Mohammed I. Abd El-Ghany ${ }^{2}$ \\ ${ }^{1}$ Department of Internal Medicine at Minit El-Nassr Central Hospital, Mansoura Ministry of Health and \\ Population, Mansoura, Egypt. \\ ${ }^{2}$ Department of Internal Medicine (Endocrinology \& Diabetes unit), Faculty of Medicine, Mansoura \\ University, Mansoura, Egypt. \\ ${ }^{3}$ Department of Clinical Pathology, Faculty of Medicine, Mansoura University, Mansoura, Egypt.
}

DOI : 10.21608/mjmu.2021.93162.1037

Submit Date: 29-08-2021

Accept Date : 15-09-2021

Available online: $30-09-2021$

\section{Keywords}

- $\mathrm{T} 2 \mathrm{DM}$

- older diabetics

- younger

diabetics

- $\mathrm{HbA1c}$

- International

Diabetes

Federation

(IDF).

\section{Abstract}

Background: Diabetes prevalence increases with age. In 2019, the IDF estimated the number of diabetic people above 65 years is 135.6 million (19.3\%), expected to be 276.2 million in 2045 , leading to a significant increase in the diabetes population of the aging societies in the next 25 years and the inevitable public health, and economic challenges this will bring. Aim: To evaluate how clinical characteristics, degree of control of glycemia, and presence of chronic complications differ between older and younger T2DM subjects. Methods: A cross-sectional study that includes 200 T2DM participants were classified into 2 groups. Group A includes 100 young subjects (<65years). Group B includes 100 old subjects (>65years). Detailed clinical history was taken. Also, HbA1c was measured. Results: Glycemic control was achieved in $46 \%$ of older diabetics vs. $37 \%$ of younger diabetics (HbA1c 7.2 vs 7.8), but statistically insignificant. Univariate analysis revealed non-obese vs. obese, DBP $\leq 85$ vs $>85$, TC level $\leq 158 \mathrm{mg} / \mathrm{dl} \mathrm{vs}>158$, and TG level $\leq 129 \mathrm{mg} / \mathrm{dl}$ vs. $>129$ were statistically significant predictors of diabetic control. Multivariate analysis revealed non-obese state $(\mathrm{OR}=2.2)$ and $\mathrm{DBP} \leq 85 \mathrm{mmHg}(\mathrm{OR}=2.1)$ were statistically significant independent predictors of the likelihood of diabetic control. The best cut-off values for DBP, TC, TG were 85, 158, 129, and the AUC were $0.593(\mathrm{p}=0.016), 0.579(\mathrm{p}=0.053), 0.574$ $(\mathrm{p}=0.072)$ respectively, to identify the optimal levels for the prediction of a controlled diabetic state. Conclusion: Control of glycemia and cardiovascular risk factors was better in the elderly T2DM subjects, however, the complications were more common among them. 


\section{INTRODUCTION}

Diabetes is a huge and rapidly growing health problem worldwide. In 2019, IDF estimated that the number of people with diabetes was 463 million and expected to be 578 million by 2030 , and 700 million by 2045. Two-thirds of people with diabetes live in urban areas, and one in five people with diabetes is above 65 years [1].

T2DM is characterized by peripheral insulin resistance and pancreatic $\beta$-cell failure, leading to major comorbidity and mortality as a result of micro and macrovascular complications [2]. Globally, T2DM represents 90\%-95\% of overall diabetic cases and still increasing [3].

The prevalence of diabetes increases with age so, the highest estimated prevalence is in people older than 65. In 2019, IDF estimated that the number of people with diabetes above 65 years is 135.6 million (19.3\%). If this trend continues, the number will be 195.2 million in 2030 and 276.2 million in 2045 , leading to a significant increase in the diabetes population of the aging societies in the next 25 years and the inevitable public health and economic challenges this will bring [1].

The strong link between age and diabetes is of great concern due to a progressive increase in life expectancy, which is likely to result in a substantial increase in the number of older people with diabetes, and a concomitant increase in the costs for the health system shortly. There is compelling evidence that diabetes in older people has differential characteristics compared with diabetes in middle-aged or earlier people [4].

Older diabetics have a similar risk for micro and macrovascular complications as younger diabetics. However, they have a more absolute higher risk for cardiovascular diseases and a higher rate of morbidity and mortality than older people without diabetes. Also, they are at higher risk for functional disabilities, polypharmacy, and common geriatric syndromes as depression, cognitive impairment, urinary incontinence, falls, and persistent pain [5].

Some older people don't have any complications and are easy to manage while others, are multi complicated and have additional severe diseases difficult to treat even in highly specialized centers [6]. The main troublesome comorbidities in the elderly are heart and kidney insufficiencies leading to a limitation in medicine prescription [7].

Blood glucose targets vary according to the patient's health status and life expectancy. According to IDF recommendations, older people who are physically fit or independent and cognitively intact should have a hemoglobin A1c (HbA1c) below 7\%, which means a rate similar to one of younger diabetics because of their long life expectancy [8].

In the view of these data, we aimed to evaluate how clinical characteristics, degree of control of glycemia, and presence of chronic complications differ between older and younger subjects with T2DM.

\section{Methods:}

\section{Patients:}

This observational, Cross-Sectional, comparative study includes 200 participants, 100 old subjects (>65years) with T2DM, and 100 young subjects (<65years) with T2DM. In all cases, both men and women were recruited from subjects attending diabetes and endocrinology outpatient clinic at Mansoura specialized medical 
hospital, Mansoura University, Egypt. The study was carried out over the period from June 2019 to June 2020. The agreement to participate in the study by informed written consent was approved by the local ethical committee at the Mansoura faculty of medicine. This research was approved by Institutional Review Board (IRB) Mansoura Faculty of Medicine, Mansoura University. The diabetic state was confirmed or excluded according to the revised American Diabetes Association criteria.

\section{The following were excluded:}

1) Subjects with Type 1 Diabetes mellitus.

2) Subjects with Gestational Diabetes.

3) Subjects who are seriously ill.

4) Subjects with other endocrinal disorders and pregnant women.

5) Subjects refuse to participate in the study.

\section{Grouping of the patients:}

The 200 participants classified into 2 groups:

- Group A: 100 young subjects (<65years) with T2DM (29 men, 71 women).

- Group B: 100 old subjects (>65years) with T2DM (45 men and 55 women).

\section{Clinical and Anthropometric Measurements:}

All participants were subjected to full medical history, anthropometric measurements as weight, height, and body mass index (BMI)), and complete clinical examination with specific reference to any micro or macrovascular complications.

Weight and height were measured using calibrated instruments after removal of heavy clothes and asking participants to take off their shoes. BMI was computed as a person's weight in kilograms divided by the square of their height in meters, like weight $/$ height ${ }^{2}\left[\mathrm{~kg} / \mathrm{m}^{2}\right]$. According to the NIH 1998 Clinical Guidelines, overweight was defined as a BMI of 25 to $29.9 \mathrm{~kg} / \mathrm{m}^{2}$ and obesity greater than $30 \mathrm{~kg} / \mathrm{m}^{2}$ [9].

All participants were evaluated regarding various microvascular complications such as diabetic retinopathy using fundus camera $\&$ retinal imaging (TRC-50DX Series). The quantitative urine albumin/creatinine ratio in the morning spot urine samples, eGFR, increased BUN, and creatinine was used for the standard diagnosis of diabetic nephropathy. Diabetic neuropathy was diagnosed based on clinical data available and EMG. Also, ECG was performed.

\section{Blood Sampling and Biochemical Measurements:}

After overnight fasting (12h), venous blood samples $(5 \mathrm{ml})$ were withdrawn from each subject via proper venipuncture technique under complete aseptic condition. A complete hemogram was performed by using an automatic blood counter (CBC Analyzer Sysmex XP300, Germany). HbA1c was measured by the ionexchange chromatography method (Biosystem co, Spain). Plasma glucose was measured by the glucose oxidase method (Cobas Integra 400 plus, Germany). Total cholesterol (TC), triglyceride (TG), and high-density lipoprotein cholesterol (HDL-C), levels were measured by spectrophotometric method (Human, Germany). Low-density lipoprotein cholesterol (LDL-C) levels were calculated using the Friedewald equation $\mathrm{LDL}=\mathrm{TC}-\mathrm{HDL}-(\mathrm{TG} / 5)$, provided that TG level not above $400 \mathrm{mg} / \mathrm{dl}$ [10]. 
Statistical analysis:

Data were entered and analyzed using IBM-SPSS software (IBM Corp. Released 2017. IBM SPSS Statistics for Windows, Version 25.0. Armonk, NY: IBM Corp.) Qualitative data were expressed as frequency $(\mathrm{N})$ and percentage $(\%)$. Quantitative data were initially tested for normality using Shapiro-Wilk's test with data being normally distributed if, $p>0.050$. The presence of significant outliers (extreme values) was tested by inspecting the boxplots. Quantitative data were expressed as mean \pm standard deviation (SD) if normally distributed, or median and interquartile range $(\mathrm{IQR})$ if not. $\mathrm{IQR}=75$ th percentile minus 25th percentile. Qualitative data were compared by the Chi-Square test. Quantitative data in two groups were compared by Independent-Samples t-Test (for normally distributed data in both groups) or its nonparametric equivalent; Mann-Whitney $U$ test if data were not normally distributed in one or both groups and/or significant outliers. The Receiver Operating Characteristic (ROC) curves were used to define the cut-points that discriminate between diabetic control vs. diabetic uncontrolled state for DBP, TC, and TG. Binary logistic regression was run to ascertain the effects of predictor variables on the likelihood that participants will exhibit diabetic control. Initially, univariate analysis was run to get crude odds ratios (COR) with their 95\% confidence interval $(95 \% \quad \mathrm{CI})$ followed by multivariate analysis to get the independent predictors with their odds ratios (OR) and 95\% CI. For any of the used tests, results were considered statistically significant if $\mathrm{p}$-value $\leq 0.050$. Appropriate charts were used to graphically present the results whenever needed including Bar chart and ROC curves.

\section{Results:}

\section{Demographic data of subjects:}

Our 200 diabetic participants were classified into two groups: Younger diabetic $(<65$ years) group included 100 (29 men, 71 women) subjects with age ranges from 32 to 64 years and the Older diabetic ( $\geq 65$ years) group included 100 (45 men and 55 women) subjects with age ranges from 65 to 84 years.

\section{Qualitative and Quantitative characteristics} between the two groups

Table (1) showed that glycemic control was achieved in $46 \%$ of older diabetics vs. $37 \%$ of younger diabetics, but this difference wasn't statistically significant. It showed that hypertension was more in older diabetics. For antihypertensive medications, monotherapy was more in younger diabetics while dual therapy was more in older diabetics. Only $4 \%$ of older diabetics were on triple therapy. The use of antiplatelet therapy was more in older diabetics. It also showed that ideal weight and overweight were more in older diabetics while obesity grades II and III were more in younger diabetics. It also showed that IHD and diabetic retinopathy were both more in older diabetics.

Also, this table showed a lower median hemoglobin A1c in older diabetics vs. younger diabetics (7.2 vs 7.8 ), but this difference wasn't statistically significant. This table showed a statistically significantly higher duration of diabetes and HDL-C in older diabetics vs. younger diabetics. 
Table (1): Qualitative and Quantitative characteristics between the two groups

\begin{tabular}{|c|c|c|c|c|c|}
\hline \multicolumn{2}{|c|}{ Characteristic } & \multirow{3}{*}{$\begin{array}{c}\begin{array}{c}\text { Younger diabetic } \\
(\mathbf{N}=\mathbf{1 0 0})\end{array} \\
63 \\
37\end{array}$} & \multirow{3}{*}{$\begin{array}{c}\begin{array}{c}\text { Older diabetic } \\
(\mathbf{N}=100)\end{array} \\
54 \\
46 \\
\end{array}$} & \multirow{3}{*}{$\begin{array}{c}\chi^{2} \\
1.668\end{array}$} & \multirow{3}{*}{$\begin{array}{c}\text { P value } \\
0.196\end{array}$} \\
\hline \multirow{2}{*}{$\begin{array}{l}\text { Glycemic } \\
\text { control }\end{array}$} & $>7 \%$ & & & & \\
\hline & $\leq 7 \%$ & & & & \\
\hline \multirow{3}{*}{$\begin{array}{l}\text { Antidiabetic } \\
\text { drugs }\end{array}$} & Oral & 44 & 45 & \multirow[t]{3}{*}{4.090} & \multirow[t]{3}{*}{0.129} \\
\hline & Insulin & 39 & 28 & & \\
\hline & Both & 17 & 27 & & \\
\hline \multirow{4}{*}{ Hypertension } & Positive & 76 & 91 & \multirow{4}{*}{$\begin{array}{c}8.165 \\
\text { FET }\end{array}$} & \multirow{4}{*}{$\begin{array}{l}0.004 \\
0.004\end{array}$} \\
\hline & Monotherapy & $46(60.5 \%) \mathrm{a}$ & $35(38.5 \%) b$ & & \\
\hline & Dual therapy & $30(39.5 \%) \mathrm{a}$ & $52(57.1 \%) b$ & & \\
\hline & Triple therapy & $0(0 \%) \mathrm{a}$ & $4(4.4 \%) \mathrm{a}$ & & \\
\hline \multicolumn{2}{|c|}{ Antiplatelet use } & 57 & 77 & 9.046 & 0.003 \\
\hline \multicolumn{2}{|l|}{ Statin use } & 43 & 42 & 0.020 & 0.886 \\
\hline \multirow{5}{*}{$\begin{array}{l}\text { BMI } \\
\text { category }\end{array}$} & Ideal & 3 & 7 & \multirow[t]{5}{*}{11.672} & \multirow[t]{5}{*}{0.020} \\
\hline & Overweight & 12 & 26 & & \\
\hline & Obese Class I & 30 & 32 & & \\
\hline & Obese Class II & 34 & 24 & & \\
\hline & Obese Class III & 21 & 11 & & \\
\hline \multirow{3}{*}{$\begin{array}{l}\text { Smoking } \\
\text { status }\end{array}$} & Current smoker & 6 & 12 & \multirow[t]{3}{*}{3.537} & \multirow[t]{3}{*}{0.171} \\
\hline & Non-smoker & 79 & 68 & & \\
\hline & Ex-smoker & 15 & 20 & & \\
\hline \multicolumn{2}{|c|}{ Ischemic Heart Disease } & 12 & 25 & 5.604 & 0.018 \\
\hline \multicolumn{2}{|c|}{ Diabetic Neuropathy } & 83 & 89 & 1.495 & 0.221 \\
\hline \multicolumn{2}{|c|}{ Diabetic Retinopathy } & 40 & 56 & 5.128 & 0.024 \\
\hline \multicolumn{2}{|c|}{ Quantitative } & $\begin{array}{c}\text { Median (Min- } \\
\text { Max) }\end{array}$ & $\begin{array}{c}\text { Median (Min- } \\
\text { Max) }\end{array}$ & $\mathbf{Z}$ & $P$ value \\
\hline \multicolumn{2}{|c|}{ Hemoglobin A1c\% } & $7.8(6.5-10.5)$ & $7.2(6.6-9.3)$ & -1.063 & 0.288 \\
\hline \multicolumn{2}{|c|}{ DM duration (years) } & $7(4-11)$ & $11(5-17.8)$ & -3.599 & $<0.001$ \\
\hline \multicolumn{2}{|l|}{ SBP (mmHg) } & $120(120-130)$ & $130(120-130)$ & -0.282 & 0.778 \\
\hline \multicolumn{2}{|l|}{ DBP (mmHg) } & $80(80-90)$ & $80(80-90)$ & -1.492 & 0.136 \\
\hline MAP (mmHg) & & $96.7(93.3-103.3)$ & $96.7(90-103.3)$ & -0.865 & 0.387 \\
\hline Total cholester & ol (mg/dL) & $173.5(140-210)$ & $183(151.5-210.8)$ & -1.239 & 0.215 \\
\hline HDL-C (mg/dI & & $40(36.3-47.5)$ & $44(39-50)$ & -2.555 & 0.011 \\
\hline LDL-C (mg/dI & & $107(70.5-135.5)$ & $113(81-138)$ & -0.646 & 0.518 \\
\hline Triglycerides ( & ng/dL) & $116(89-159.8)$ & $121.5(89.3-161.8)$ & -0.181 & 0.857 \\
\hline Serum creatini & ne (mg/dL) & $0.8(0.7-1.0)$ & $0.8(0.6-0.9)$ & -1.486 & 0.137 \\
\hline eGFR $(\mathrm{ml} / \mathrm{min}$ & $\left.1.73 \mathrm{~m}^{2}\right)$ & $83(68-112)$ & $102(70.8-129.8)$ & -1.549 & 0.121 \\
\hline Albumin creat & nine ratio $(\mathrm{mg} / \mathrm{g})$ & $33(24.3-53)$ & $37(23-82)$ & -0.298 & 0.765 \\
\hline
\end{tabular}

Data are expressed as $\mathrm{N}$ and compared by Chi-Square test. For antihypertensive use, data expressed as $\mathrm{N}(\%)$ and compared by Fisher's exact test (FET). Also, data are expressed as Median (IQR) and compared by MannWhitney U-test.

Qualitative and Quantitative characteristics between the two glycemic control groups

There was no statistically significant difference in all qualitative parameters between controlled and uncontrolled groups as shown in
Table (2) except for obesity which was statistically significantly more frequent in uncontrolled vs. controlled group $(\mathrm{p}=0.001)$.

Also, Table (2) showed a statistically significantly higher DBP, MAP, and BMI in the 
uncontrolled vs. controlled group ( $\mathrm{p}=0.017,0.044$,

$0.005)$, respectively. It also showed lower values

for age, and higher values for serum TC and TG in

the uncontrolled vs. controlled group. However, these differences didn't achieve a statistical significance. There was no statistically significant difference as regards DM duration, SBP, HDL-C, LDL-C, serum creatinine, eGFR, and ACR.

Table (2): Qualitative and Quantitative characteristics between the two glycemic control groups

\begin{tabular}{|c|c|c|c|c|c|}
\hline \multicolumn{2}{|c|}{ Characteristic } & \multirow{2}{*}{$\begin{array}{c}\begin{array}{c}\text { A1c }>7 \% \\
(\mathbf{N}=117)\end{array} \\
54(46.2 \%)\end{array}$} & \multirow{2}{*}{$\begin{array}{c}\begin{array}{c}\mathbf{A} 1 \mathrm{c} \leq 7 \% \\
(\mathbf{N}=\mathbf{8 3})\end{array} \\
46(55.4 \%)\end{array}$} & \multirow{3}{*}{$\chi^{2}$} & \multirow{2}{*}{$\begin{array}{c}\text { P value } \\
0.196\end{array}$} \\
\hline Age groups & $\geq 65 \%$ & & & & \\
\hline & $<65 \%$ & $63(53.8 \%)$ & $37(44.6 \%)$ & & \\
\hline \multirow{3}{*}{$\begin{array}{l}\text { Antidiabetic } \\
\text { drugs }\end{array}$} & Oral & $49(41.9 \%)$ & $40(48.2 \%)$ & \multirow[t]{3}{*}{0.953} & \multirow[t]{3}{*}{0.621} \\
\hline & Insulin & $40(34.2 \%)$ & $27(32.5 \%)$ & & \\
\hline & Both & $28(23.9 \%)$ & $16(19.3 \%)$ & & \\
\hline \multirow[t]{4}{*}{ Hypertension } & Positive & $100(85.5 \%)$ & $67(80.7 \%)$ & \multirow{4}{*}{$\begin{array}{c}0.794 \\
\text { FET }\end{array}$} & \multirow{4}{*}{$\begin{array}{l}0.373 \\
0.912\end{array}$} \\
\hline & Monotherapy & $48(48.0 \%)$ & $33(49.3 \%)$ & & \\
\hline & Dual therapy & $50(50.0 \%)$ & $32(47.8 \%)$ & & \\
\hline & Triple therapy & $2(2.0 \%)$ & $2(3 \%)$ & & \\
\hline \multicolumn{2}{|l|}{ Antiplatelet use } & $81(69.2 \%)$ & $53(63.9 \%)$ & 0.635 & 0.426 \\
\hline \multicolumn{2}{|l|}{ Statin use } & $50(42.7 \%)$ & $35(42.2 \%)$ & 0.006 & 0.936 \\
\hline \multirow{5}{*}{ BMI category } & Ideal & $6(5.1 \%) \mathrm{a}$ & $3(3.6 \%) \mathrm{a}$ & \multirow[t]{5}{*}{ FET } & \multirow[t]{5}{*}{0.005} \\
\hline & Overweight & $12(10.3 \%) \mathrm{a}$ & $26(31.3 \%) \mathrm{b}$ & & \\
\hline & Obese Class I & $39(33.3 \%) \mathrm{a}$ & $24(28.9 \%) \mathrm{a}$ & & \\
\hline & Obese Class II & $40(34.2 \%) \mathrm{a}$ & $18(21.7 \%) \mathrm{a}$ & & \\
\hline & Obese Class III & $20(17.1 \%) \mathrm{a}$ & $12(14.5 \%) \mathrm{a}$ & & \\
\hline \multirow[t]{2}{*}{ Obesity } & Non-obese & $18(15.4 \%)$ & $29(34.9 \%)$ & \multirow[t]{2}{*}{10.328} & \multirow[t]{2}{*}{0.001} \\
\hline & Obese & $99(84.6 \%)$ & $54(65.1 \%)$ & & \\
\hline \multirow{3}{*}{ Smoking status } & Current smoker & $9(7.7 \%)$ & $9(10.8 \%)$ & \multirow[t]{3}{*}{5.438} & \multirow[t]{3}{*}{0.066} \\
\hline & Non-smoker & $93(79.5 \%)$ & $54(65.1 \%)$ & & \\
\hline & Ex-smoker & $15(12.8 \%)$ & $20(24.1 \%)$ & & \\
\hline \multicolumn{2}{|c|}{ Ischemic Heart Disease } & $19(16.2 \%)$ & $18(21.7 \%)$ & 0.956 & 0.328 \\
\hline \multicolumn{2}{|c|}{ Diabetic Neuropathy } & $104(88.9 \%)$ & $68(81.9 \%)$ & 1.954 & 0.162 \\
\hline \multicolumn{2}{|c|}{ Diabetic Retinopathy } & $58(49.6 \%)$ & $38(45.8 \%)$ & 0.279 & 0.597 \\
\hline \multicolumn{2}{|l|}{ Quantitative } & Median (Min-Max) & Median (Min-Max) & $\mathbf{Z}$ & $P$ value \\
\hline \multicolumn{2}{|l|}{ Age (years) } & $62(51-67.5)$ & $66(56-68)$ & -1.793 & 0.073 \\
\hline DM duration (y & & $9(4-13.5)$ & $8(5-14)$ & -0.232 & 0.816 \\
\hline SBP (mmHg) & & $130(120-130)$ & $130(120-130)$ & -1.214 & 0.225 \\
\hline DBP (mmHg) & & $80(80-90)$ & $80(70-90)$ & -2.378 & 0.017 \\
\hline MAP (mmHg) & & $96.7(93.3-103.3)$ & $93.3(90-103.3)$ & -2.011 & 0.044 \\
\hline BMI (kg/m2) & & $35(31-38.5)$ & $32(29-37)$ & -2.803 & 0.005 \\
\hline Total cholestero & $\mathrm{ng} / \mathrm{dL})$ & $184(154-214)$ & $176(136-203)$ & -1.892 & 0.058 \\
\hline HDL-C (mg/dL) & & $41(37-50)$ & $41(38-49)$ & -0.238 & 0.812 \\
\hline LDL-C (mg/dL) & & $112(71.3-142.5)$ & $103.5(72-134)$ & -0.971 & 0.332 \\
\hline Triglycerides (m & IL) & $123(92.5-169)$ & $112(84-148)$ & -1.785 & 0.074 \\
\hline Serum creatinin & $\mathrm{mg} / \mathrm{dL}$ ) & $0.8(0.6-1.0)$ & $0.8(0.6-0.9)$ & -0.859 & 0.390 \\
\hline eGFR $(\mathrm{ml} / \mathrm{min} / 1$ & m2) & $94(67.5-121)$ & $88(70-118)$ & -0.859 & 0.848 \\
\hline Albumin creatin & ratio $(\mathrm{mg} / \mathrm{g})$ & $32(24-63)$ & $37(24-66)$ & -0.608 & 0.543 \\
\hline
\end{tabular}

Data are expressed as N and compared by Chi-Square test. For antihypertensive use, data expressed as N (\%) and compared by Fisher's exact test (FET). Also, data are expressed as Median (IQR) and compared by Mann-Whitney U-test.

\section{Qualitative and Quantitative characteristics in elderly diabetics}

Table (3), showing no statistically significant difference in all qualitative and quantitative parameters between controlled and uncontrolled elderly diabetics groups except for smoking status, obesity which was statistically significantly more frequent in uncontrolled vs. 
controlled group and sex where female patients are more frequent in the uncontrolled group. Also, uncontrolled vs. controlled elderly diabetics groups.

BMI was statistically significantly higher among

Table (3): Qualitative and Quantitative characteristics in elderly diabetics

\begin{tabular}{|c|c|c|c|c|c|}
\hline \multicolumn{2}{|c|}{ Characteristic } & \multirow{2}{*}{$\begin{array}{c}\begin{array}{c}\text { A1c }>\text { 7\% } \\
(\mathbf{N}=\mathbf{5 4})\end{array} \\
19(35.2 \%)\end{array}$} & \multirow{2}{*}{$\begin{array}{c}\mathrm{A} 1 \mathrm{c} \leq 7 \% \\
(\mathbf{N}=\mathbf{4 6}) \\
26(56.5 \%)\end{array}$} & \multirow{3}{*}{$\begin{array}{c}\chi^{2} \\
4.569\end{array}$} & \multirow{2}{*}{\begin{tabular}{|c|} 
P value \\
0.033
\end{tabular}} \\
\hline \multirow[t]{2}{*}{ Sex } & Male & & & & \\
\hline & Female & $35(64.8 \%)$ & $20(43.5 \%)$ & & \\
\hline \multirow[t]{3}{*}{ Antidiabetic drugs } & Oral & $22(40.7 \%)$ & $23(50 \%)$ & \multirow[t]{3}{*}{1.349} & \multirow[t]{3}{*}{0.510} \\
\hline & Insulin & $15(27.8 \%)$ & $13(28.3 \%)$ & & \\
\hline & Both & $17(31.5 \%)$ & $10(21.7 \%)$ & & \\
\hline \multirow[t]{4}{*}{ Hypertension } & Positive & $49(90.7 \%)$ & $42(42.9 \%)$ & \multirow{4}{*}{$\begin{array}{l}\text { FET } \\
\text { FET }\end{array}$} & \multirow{4}{*}{$\begin{array}{l}1.000 \\
0.717\end{array}$} \\
\hline & Monotherapy & $17(34.7 \%)$ & $18(49.3 \%)$ & & \\
\hline & Dual therapy & $30(61.2 \%)$ & $22(52.4 \%)$ & & \\
\hline & Triple therapy & $2(4.1 \%)$ & $2(4.8 \%)$ & & \\
\hline \multicolumn{2}{|l|}{ Antiplatelet use } & $45(83.3 \%)$ & $32(69.6 \%)$ & 2.659 & 0.103 \\
\hline \multicolumn{2}{|l|}{ Statin use } & $23(42.6 \%)$ & $19(41.3 \%)$ & 0.017 & 0.896 \\
\hline \multirow{5}{*}{ BMI category } & Ideal & $4(7.4 \%) \mathrm{a}$ & $2(4.3 \%) \mathrm{a}$ & \multirow[t]{5}{*}{ FET } & \multirow[t]{5}{*}{0.001} \\
\hline & Overweight & $5(9.3 \%) \mathrm{a}$ & $21(45.7 \%) \mathrm{b}$ & & \\
\hline & Obese Class I & $24(44.4 \%) \mathrm{a}$ & $9(19.6 \%) \mathrm{a}$ & & \\
\hline & Obese Class II & $15(27.8 \%) \mathrm{a}$ & $9(19.6 \%) \mathrm{a}$ & & \\
\hline & Obese Class III & $6(11.1 \%) \mathrm{a}$ & $5(10.9 \%) \mathrm{a}$ & & \\
\hline \multirow[t]{2}{*}{ Obesity } & Non-obese & $9(16.7 \%)$ & $23(50 \%)$ & \multirow[t]{2}{*}{12.684} & \multirow[t]{2}{*}{$<0.001$} \\
\hline & Obese & $45(83.3 \%)$ & $23(50 \%)$ & & \\
\hline \multirow[t]{3}{*}{ Smoking status } & Current smoker & $5(9.3 \%)$ & $7(15.2 \%)$ & \multirow[t]{3}{*}{7.707} & \multirow[t]{3}{*}{0.021} \\
\hline & Non-smoker & $43(79.6 \%)$ & $25(54.3 \%)$ & & \\
\hline & Ex-smoker & $6(11.1 \%)$ & $14(30.4 \%)$ & & \\
\hline \multicolumn{2}{|c|}{ Ischemic Heart Disease } & $11(20.4 \%)$ & $14(30.4 \%)$ & 1.342 & 0.247 \\
\hline \multicolumn{2}{|l|}{ Diabetic Neuropathy } & $49(90.7 \%)$ & $40(87 \%)$ & 0.363 & 0.547 \\
\hline \multicolumn{2}{|l|}{ Diabetic Retinopathy } & $32(59.3 \%)$ & $24(52.2 \%)$ & 0.506 & 0.477 \\
\hline \multicolumn{2}{|c|}{ Quantitative } & Median (Min-Max) & Median (Min-Max) & $\mathbf{Z}$ & $P$ value \\
\hline & Age (years) & $68(66-70.3)$ & $67.5(76-72)$ & -0.759 & 0.448 \\
\hline DM duration (years & & $11.5(5-18)$ & $10(5-17.5)$ & -0.010 & 0.992 \\
\hline SBP (mmHg) & & $130(120-130)$ & $130(120-130)$ & -0.025 & 0.980 \\
\hline DBP (mmHg) & & $80(80-90)$ & $80(70-90)$ & -1.637 & 0.102 \\
\hline MAP (mmHg) & & $96.7(93.3-103.3)$ & $93.3(90-103.3)$ & -0.981 & 0.326 \\
\hline BMI (kg/m2) & & $33(30.8-37.3)$ & $29(27-36)$ & -2.382 & 0.017 \\
\hline Total cholesterol (m & dL) & $191.5(158.3-219.5)$ & $178(141.2-205)$ & -1.639 & 0.101 \\
\hline HDL-C (mg/dL) & & $44(39.8-50)$ & $42.5(38.7-50)$ & -0.499 & 0.618 \\
\hline LDL-C (mg/dL) & & $113(84-143.5)$ & $112.5(77.8-132.2)$ & -0.923 & 0.356 \\
\hline Triglycerides (mg/d & & $130(91.3 .5-170.8)$ & $110.5(87-151)$ & -1.203 & 0.229 \\
\hline Serum creatinine (n & $/ \mathrm{dL})$ & $0.75(0.6-1.0)$ & $0.8(0.6-0.9)$ & -0.098 & 0.922 \\
\hline eGFR $(\mathrm{ml} / \mathrm{min} / \mathbf{1 . 7 3}$ & & $101(64.3-127.5)$ & $102(76-139.5)$ & -0.827 & 0.408 \\
\hline Albumin creatinine & tio $(\mathrm{mg} / \mathrm{g})$ & $37(23-84.2)$ & $35(22-82)$ & -0.478 & 0.633 \\
\hline
\end{tabular}

Data are expressed as $\mathrm{N}$ and compared by Chi-Square test. For antihypertensive use, data expressed as $\mathrm{N}$ (\%) and compared by Fisher's exact test (FET). Also, data are expressed as Median (IQR) and compared by Mann-Whitney U-test.

\section{Predictors of the likelihood of controlled} diabetic state

Table (4), showing the results of binary logistic regression, which was run to ascertain the effects of age $\geq 65$ years, non-obese state, $\mathrm{DBP} \leq 85$
mmHg, TC level $\leq 158 \mathrm{mg} / \mathrm{dl}$, and TG level $\leq 129$ $\mathrm{mg} / \mathrm{dl}$ on the likelihood that participants will achieve diabetic control. Univariate analysis revealed that non-obese vs. obese, DBP $\leq 85$ vs $>85$, TC level $\leq 158 \mathrm{mg} / \mathrm{dl}$ vs $>158$, and TG level 
$\leq 129 \mathrm{mg} / \mathrm{dl}$ vs. $>129$ were statistically significant predictors of diabetic control. Age wasn't a statistically significant predictor. Multivariate analysis was run and revealed that only non-obese state and DBP $\leq 85 \mathrm{mmHg}$ were statistically significant independent predictors of the likelihood of diabetic control. Non-obese diabetics have 2.2 times higher odds than obese diabetics to exhibit diabetic control and diabetics with DBP $\leq 85$ mmHg have 2.1 times higher odds than diabetics with DBP> $85 \mathrm{mmHg}$ to exhibit diabetic control. This model was statistically significant $\left(\chi^{2}[5]=\right.$ $22.22, \mathrm{P}<0.001)$ and correctly classified $66 \%$ of cases.

Table (4): Predictors of the likelihood of controlled diabetic state

\begin{tabular}{|c|c|c|c|c|c|c|c|}
\hline \multicolumn{2}{|c|}{ Predictor } & \multicolumn{3}{|c|}{ Univariate analysis } & \multicolumn{3}{|c|}{ Multivariate analysis } \\
\hline & & $P$ value & COR & $95 \% \mathrm{CI}$ & P value & COR & $95 \% \mathrm{CI}$ \\
\hline \multirow[t]{2}{*}{ Age } & $<65$ years & \multirow[t]{2}{*}{0.197} & $\mathrm{R}$ & $\mathrm{R}$ & \multirow[t]{2}{*}{0.493} & $\mathrm{R}$ & $\mathrm{R}$ \\
\hline & $\geq 65$ years & & 1.45 & $0.82-2.55$ & & 1.24 & $0.67-2.29$ \\
\hline \multirow[t]{2}{*}{ Obesity status } & Obese & \multirow[t]{2}{*}{0.002} & $\mathrm{R}$ & $\mathrm{R}$ & \multirow[t]{2}{*}{0.030} & $\mathrm{R}$ & $\mathrm{R}$ \\
\hline & Non-obese & & 2.95 & $1.5-5.8$ & & 2.2 & $1.1-4.5$ \\
\hline \multirow[t]{2}{*}{ DBP (mmHg) } & $>85$ & \multirow[t]{2}{*}{0.006} & $\mathrm{R}$ & $\mathrm{R}$ & \multirow[t]{2}{*}{0.020} & $\mathrm{R}$ & $\mathrm{R}$ \\
\hline & $\leq 85$ & & 2.36 & $1.27-4.36$ & & 2.1 & $1.1-4.1$ \\
\hline \multirow[t]{2}{*}{ TC (mg/dl) } & $>158$ & \multirow[t]{2}{*}{0.030} & $\mathrm{R}$ & $\mathrm{R}$ & \multirow[t]{2}{*}{0.148} & $\mathrm{R}$ & $\mathrm{R}$ \\
\hline & $\leq 158$ & & 1.94 & $1.1-3.5$ & & 1.6 & $0.8-3.1$ \\
\hline \multirow[t]{2}{*}{ TG (mg/dl) } & $>129$ & \multirow[t]{2}{*}{0.020} & $\mathrm{R}$ & $\mathrm{R}$ & \multirow[t]{2}{*}{0.102} & $\mathrm{R}$ & $\mathrm{R}$ \\
\hline & $\leq 129$ & & 2.13 & $1.1-3.6$ & & 1.7 & $0.9-3.2$ \\
\hline
\end{tabular}

Performance characteristics of some parameters for prediction of controlled diabetic state

In Table (5), ROC analysis was conducted to identify the optimal levels for the prediction of a controlled diabetic state. DBP best cut-off values

Table (5): Performance characteristics of some parameters for prediction of controlled diabetic state.

\begin{tabular}{|c|c|c|c|c|c|}
\hline & AUC & $\boldsymbol{p}$ & $\begin{array}{c}\text { Cut } \\
\text { off }\end{array}$ & $\begin{array}{c}\text { Sensitivity } \\
(\boldsymbol{\%})\end{array}$ & $\begin{array}{c}\text { Specificity } \\
(\boldsymbol{\%})\end{array}$ \\
\hline DBP (mmHg) & 0.593 & 0.016 & 85.0 & 74.7 & 44.4 \\
\hline TC (mg/dl) & 0.579 & 0.053 & 158 & 42.2 & 72.6 \\
\hline TG (mg/d) & 0.574 & 0.072 & 129 & 68.7 & 47.9 \\
\hline
\end{tabular}

were 85.0, with the area under the curve (AUC) was $0.593(\mathrm{p}=0.016)$. TC best cut-off values were 158, with the area under the curve (AUC) was $0.579(\mathrm{p}=0.053)$. TG best cut-off values were 129, with the area under the curve (AUC) was 0.574 $(\mathrm{p}=0.072)$. 


\section{Discussion:}

Diabetes is a chronic disease that harms different organs due to the negative effect of hyperglycemia and requires ongoing management. There is evidence that diabetic complications, mortality, and hospitalization are more prevalent among older people with diabetes [11].

The relationship between age and glycemic control has been reported to be questionable. Some studies have reported a high prevalence of poor control in the elderly, some studies point to no effect of age on metabolic control, to better glycemic control among older people, and even point to an inverse relationship between age and glycemic control [12].

On the other hand, worse glycemic control and lipid profile, higher prevalence of obesity and additional cardiovascular risk factors, remarkable increase in the risk of cardiovascular disease, higher rate of death from the hyperglycemic crisis, were reported among younger type 2 diabetic subjects by some studies [13].

Older people with T2DM are a growing population that accounts for approximately $50 \%$ of all cases of diabetes in adults and have differential characteristics, requiring tailored management approaches [5].

The main objective of this Cross-Sectional study was to evaluate how clinical characteristics, degree of control of glycemia, and presence of chronic complications differ between older and younger subjects with T2DM.

In our current study, the glycemic control was achieved in $46 \%$ of older diabetics vs. $37 \%$ of younger diabetics, with lower median $\mathrm{HbA1c}$ in older diabetics vs. younger diabetics (7.2 vs 7.8), but this difference wasn't statistically significant. However, the duration of diabetes was statistically significantly higher in older diabetics vs. younger diabetics.

These results came in agreement with AlMukhtar et al. who showed that younger diabetic subjects have a significantly elevated level of HbA1c compared with older diabetic subjects [14]. Similar results were obtained by Shamshigaran et al. who noted that poor glycemic control was detected in $70.4 \%$ of the younger age group and $63.0 \%$ of the older age group with no statistical significance [12].

In the same context, Barrot-de la Puente et al. reported that the percentage of subjects with fair glycemic control $(\mathrm{HbAlc} \leq 7 \%)$ was significantly higher among older age groups in both genders $(\mathrm{p}<0.001)$ despite longer diabetes duration in older age groups [13]. Similarly, some studies reported better glycemic control among the older age groups $[15,16]$.

In our current study, hypertension was more in older diabetics. For antihypertensive medications, monotherapy was more in younger diabetics while dual therapy was more in older diabetics. Only $4 \%$ of older diabetics were on triple therapy. The use of antiplatelet therapy was more in older diabetics. It also showed that IHD and diabetic retinopathy were both more in older diabetics. HDL-C level was statistically significantly higher in older diabetics vs. younger diabetics. 
Such results agreed with Shamshigaran et

al. who reported that hypertension was more significantly frequent among older diabetic patients compared to younger diabetic patients. Moreover, complications were higher among the elderly compared to younger participants $(57.1 \%$ vs. $45.2 \%$. $\mathrm{P}=0.003$ ). The frequencies of complications in their study according to the patient's clinic record were neuropathy (34.9\%), retinopathy (16\%), nephropathy (5.9\%), stroke $(1.6 \%)$, and cardiovascular disease (6.1\%) [12].

In contrary to these results, Al-Mukhtar et al. stated that the younger diabetic subjects were found to have a significantly longer duration of disease and a significant increase in SBP and DBP in comparison with the older subjects $(\mathrm{p}<0.005)$. Also, there was a significant elevation of TC, TG, and LDL-C in younger diabetic compared to older diabetic [14].

In our current study, ideal weight and overweight were more in older diabetics while obesity class II and III were more in younger diabetics.

This was in agreement with Al-Mukhtar et al. who reported that the BMI of the younger diabetic subjects was significantly higher than in older subjects. Significant increase of overweight and obese frequency in younger diabetic subjects compared to older diabetic subjects [14]. Also, Sattar et al. stated that there was a significant reduction of BMI in older age groups compared to younger age groups [17].

In contrast to these results, Shamshigaran et al. documented that there was no significant difference regarding BMI among both younger and older diabetic subjects [12].

As regards qualitative characteristics between the two glycemic control groups, our current study revealed that no statistically significant difference in all qualitative parameters between controlled and uncontrolled groups except for obesity which was statistically significantly more frequent in the uncontrolled vs. controlled group.

Such results agreed with Haghighatpanah et al. who stated that subjects with poor glycemic control $(\mathrm{HbA} 1 \mathrm{c}>7 \%)$ were more obese than subjects with good glycemic control (HbA1c<7\%) [18]. Also, Anioke et al. reported that waist-hip ratio (WHR) was significantly higher among subjects with poor glycemic control (HbA1c>7\%) than subjects with good glycemic control $(\mathrm{HbA} 1 \mathrm{c}<7 \%)$ [19].

Regarding quantitative characteristics between the two glycemic control groups, our current study revealed a statistically significantly higher DBP, MAP, and BMI in uncontrolled vs. controlled groups. It also showed lower values for age and higher values for serum TC and TG in the uncontrolled vs. controlled group. However, these differences didn't achieve a statistical significance. There was no statistically significant difference as regards DM duration, SBP, HDL-C, LDL-C, serum creatinine, eGFR, and ACR.

A study done by Anioke et al. showed no significant difference regarding TC and HDL-C levels among both groups with good or poor glycemic control. However, the poor glycemic control group (HbA1c>7\%) had significantly 
higher systolic blood pressure and LDL-C levels compared with the good glycemic control group $(\mathrm{HbA} 1 \mathrm{c}<7 \%)$ [19].

Another study done by Alzahrani et al. noted that no significant difference regarding TC, HDL-C, LDL-C levels, and age among subjects with and without glycemic control [20].

Regarding Predictors of the likelihood of controlled diabetic state, in our current study multivariate analysis was run and revealed that only non-obese state and $\mathrm{DBP} \leq 85 \mathrm{mmHg}$ were statistically significant independent predictors of the likelihood of diabetic control. Non-obese diabetics have 2.2 times higher odds than obese diabetics to exhibit diabetic control and diabetics with $\mathrm{DBP} \leq 85 \mathrm{mmHg}$ have 2.1 times higher odds than diabetics with $\mathrm{DBP}>85 \mathrm{mmHg}$ to exhibit diabetic control.

Sazlina et al. in their study reported that patient characteristics associated with poor glycemic control were younger age group $(\mathrm{OR}=1.96), \mathrm{T} 2 \mathrm{DM}$ duration $>10$ years $(\mathrm{OR}=1.75)$, elevated $\mathrm{BP}(\mathrm{OR}=1.10)$, LDL-C $(\mathrm{OR}=1.48)$, and $\mathrm{TG}(\mathrm{OR}=1.61)[21]$.

Mobula et al. demonstrated that male gender $(\mathrm{OR}=0.66$, increasing age $(\mathrm{OR}=0.97)$ and dual diagnosis of diabetes and hypertension $(\mathrm{OR}=0.69)$ had positive associations with good glycemic control. While, duration of diabetes diagnosis $(\mathrm{OR}=1.04)$, the absence of the Ghana National Health Insurance Scheme (OR=1.41), and the number of diabetes medicines $(\mathrm{OR}=1.73)$ were adversely associated with poor glycemic control [22].
The results of multivariate analysis in the Haghighatpanah et al. study showed that females $(\mathrm{OR}=2.07)$, subjects $<65$ years old $(\mathrm{OR}=1.67)$, abnormal HDL-C level (OR=1.72), duration of diabetes $>10$ years, and types of diabetes medications, were all significantly associated with poor glycemic control [18].

Khanthaboon et al. stated that diabetic subjects with secondary school completed or more were 2.85 times more likely to control $\mathrm{HbA1C}$ than those with an education level $\leq$ primary school $(\mathrm{OR}=2.85 ; 95 \% \mathrm{CI}=1.17-6.98)$. Subjects with insulin therapy controlled their HbA1C level better than other groups $(\mathrm{OR}=3.04 ; 95 \% \mathrm{Cl}=1.10$ 8.35; $\mathrm{p}=0.031$ ) [23].

Regarding performance characteristics of some parameters for prediction of controlled diabetic state, the present study revealed DBP best cut-off values were 85.0, with the area under the curve (AUC) was 0.593 ( $\mathrm{p}=0.016$ ). TC best cut-off values were 158 , with the area under the curve (AUC) was $0.579(\mathrm{p}=0.053)$. TG best cut-off values were 129 , with the area under the curve (AUC) was $0.574(\mathrm{p}=0.072)$.

To our knowledge, no other study assesses the ROC curve of any of the previous parameters for the prediction of a controlled diabetic state.

\section{Conclusion:}

Glycemic control was achieved in $46 \%$ of older diabetics vs. $37 \%$ of younger diabetics (mean HbA1c 7.2 vs 7.8), but this difference wasn't statistically significant. Multivariate analysis showed that non-obese state $(\mathrm{OR}=2.2$; $95 \% \mathrm{CI}=1.1-4.5 ; \quad \mathrm{p}=0.030)$ and $\mathrm{DBP} \leq 85 \mathrm{mmHg}$ $(\mathrm{OR}=2.1 ; \quad 95 \% \mathrm{Cl}=1.1-4.1 ; \quad \mathrm{p}=0.020) \quad$ were 
statistically significant independent predictors of the likelihood of diabetic control. The best cut-off values for DBP, TC, TG were 85, 158, 129, respectively, and the AUC were $0.593(\mathrm{p}=0.016)$, $0.579(\mathrm{p}=0.053), 0.574(\mathrm{p}=0.072)$ respectively, to identify the optimal levels for the prediction of a controlled diabetic state. So, our study proposes that control of glycemia and cardiovascular risk factors was better among the elderly subjects with T2DM, however, the complications were more common among them.

\section{Abbreviations:}

IDF: International Diabetes Federation; T2DM: type 2 diabetes mellitus; CVD: cardiovascular disease; BMI: Body mass index; HbA1c: glycosylated hemoglobin; TC: total cholesterol; TG: triglyceride; HDL-C: high-density lipoprotein cholesterol; LDL-C: low-density lipoprotein cholesterol; SBP: systolic blood pressure; DBP: diastolic blood pressure; MAP: mean arterial pressure; IHD: ischemic heart disease; ACR: albumin to creatinine ratio; eGFR: estimated glomerular filtration rate; AUC: area under curve; OR: odds ratios; COR: crude odds ratios; IQR: interquartile range; CI: confidence interval; ROC: Receiver Operating Characteristic.

\section{References:}

1) Sinclair A, Saeedi P, Kaundal A, Karuranga $S$, Malanda B, Williams R. Diabetes and global ageing among 65-99-year-old adults: Findings from the International Diabetes Federation Diabetes Atlas, $9^{\text {th }}$ edition. Diabetes Res Clin Pract. 2020 Apr;162:108078.
2) American Diabetes Association. Diagnosis and classification of diabetes mellitus. Diabetes Care. 2014:37(Suppl. 1):S81-S90.

3) Hegazi R, El-Gamal M, Abdel-Hady N, Hamdy O. Epidemiology of and Risk Factors for Type 2 Diabetes in Egypt. Ann Glob Health. 2015:81(6):814-20.

4) Sinclair AJ, Paolisso G, Castro M, BourdelMarchasson I, Gadsby R, Rodriguez Mañas L; European Diabetes Working Party for Older People. European Diabetes Working Party for Older People 2011 clinical guidelines for type 2 diabetes mellitus. Executive summary. Diabetes Metab. 2011 Nov;37 Suppl 3:S27-38.

5) Kirkman MS, Briscoe VJ, Clark N, Florez H, Haas LB, Halter JB, et al. Consensus Development Conference on Diabetes and Older Adults. Diabetes in older adults: a consensus report. J Am Geriatr Soc. 2012 Dec;60(12):2342-56.

6) Cheung KK, Luk AO, So WY, Ma RC, Kong AP, Chow FC, et al. Testosterone level in men with type 2 diabetes mellitus and related metabolic effects: A review of current evidence. J Diabetes Investig. 2015 Mar;6(2):112-23.

7) Reiner M, Niermann C, Jekauc D, Woll A. Long-term health benefits of physical activity-a systematic review of longitudinal studies. BMC Public Health. 2013 Sep 8;13:813.

8) Dunning $T$, Sinclair A, Colagiuri S. New IDF Guideline for managing type 2 diabetes in older people. Diabetes Res Clin Pract. 2014 Mar;103(3):538-40. 
9) Clinical Guidelines on the Identification, Evaluation, and Treatment of Overweight and Obesity in Adults--The Evidence Report. National Institutes of Health. Obes Res. 1998 Sep;6 Suppl 2:51S-209S. Erratum in: Obes Res 1998 Nov;6(6):464.

10) Friedewald WT, Levy RI, Fredrickson DS. Estimation of the concentration of low-density lipoprotein cholesterol in plasma, without use of the preparative ultracentrifuge. Clin Chem; 1972; 18:499-502.

11) Ki M, Baek S, Yun YD, Kim N, Hyde M, Na B. Age-related differences in diabetes care outcomes in Korea: a retrospective cohort study. BMC Geriatric. 2014 Oct 16;14:111.

12) Shamshirgaran $S$, Mamaghanian $A$, Aliasgarzadeh A, Aiminisani N, IranparvarAlamdari M, Ataie J. Age differences in diabetes-related complications and glycemic control. BMC Endocr Disord. 2017;17:25.

13) Barrot-de la Puente J, Mata-Cases M, FranchNadal J, Mundet-Tuduri X, Casellas A, Fernandez-Real JM, et al. Older type 2 diabetic patients are more likely to achieve glycaemic and cardiovascular risk factors targets than younger patients: analysis of a primary care database. Intern J Clin Pract. 2015;69(12):1486-95.

14) Al-Mukhtar SB, Fadhil NN, Hanna BE. General and gender characteristics of type 2 diabetes mellitus among the younger and older age groups. Oman Med J. 2012 Sep;27(5):37582.
15) Chiu CJ, Wray LA. Factors predicting glycemic control in middle-aged and older adults with type 2 diabetes. Prevent Chron Dis. 2010;7(1):A08.

16) Toh MPHS, Wu CX, Leong HSS. Association of younger age with poor glycemic and cholesterol control in Asians with type 2 Diabetes Mellitus in Singapore. J Endocrinol Metab. 2011;1(1):27-37.

17) Sattar N, Rawshani A, Franzén $S$, Rawshani A, Svensson AM, Rosengren A, et al. Age at Diagnosis of Type 2 Diabetes Mellitus and Associations With Cardiovascular and Mortality Risks. Circulation. 2019 May 7;139(19):2228-2237.

18) Haghighatpanah M, Nejad ASM, Haghighatpanah M, Thunga G, Mallayasamy S. Factors that Correlate with Poor Glycemic Control in Type 2 Diabetes Mellitus Patients with Complications. Osong Public Health Res Perspect. 2018 Aug;9(4):167-174.

19) Anioke IC, Ezedigboh AN, Dozie-Nwakile OC, Chukwu IJ, Kalu PN. Predictors of poor glycemic control in adult with type 2 diabetes in South-Eastern Nigeria. Afr Health Sci. 2019 Dec;19(4):2819-2828.

20) Alzahrani SH, Baig M, Aashi MM, Al-Shaibi FK, Alqarni DA, Bakhamees WH. Association between glycated hemoglobin (HbA1c) and the lipid profile in patients with type 2 diabetes mellitus at a tertiary care hospital: a retrospective study. Diabetes Metab Syndr Obes. 2019 Aug 29;12:1639-1644. 
21) Sazlina SG, Mastura I, Cheong AT, Bujang Mohamad A, Jamaiyah H, Lee PY, et al. Predictors of poor glycaemic control in older patients with type 2 diabetes mellitus. Singapore Med J. 2015 May;56(5):284-90.

22) Mobula LM, Sarfo FS, Carson KA, Burnham G, Arthur L, Ansong D, et al. Predictors of glycemic control in type-2 diabetes mellitus: Evidence from a multicenter study in Ghana. Translational Metabolic Syndrome Research. 2018 Dec;1:1-8.
23) Khanthaboon $P$, Kumbudda $T$, Poonsuk $P$, Taengthonglang C, Saramunee K, Phimarn W. Factors Associated with Quality of Life and Glycemic Control in Diabetes Mellitus Type 2 Patients in Sangkha Hospital, Surin Province, Thailand. Journal of Young Pharmacists. 2020 Jun; 12(2):149-152 